\title{
Ionospheric perturbations observed by the low altitude satellite DEMETER and possible relation with seismicity
}

\author{
Y. Hobara ${ }^{1,2)}$, R. Nakamura ${ }^{1)}$, M. Suzuki ${ }^{1)}$, M. Hayakawa ${ }^{2,3)}$, and M. Parrot ${ }^{4)}$ \\ ${ }^{1}$ The University of Electro-Communications (UEC), Graduate School of Informatics and Engineering, 1-5-1 \\ Chofugaoka, Chofu Tokyo, 182-8585 Japan \\ ${ }^{2}$ UEC, Research Station on Seismo-Electromagnetics, Chofu Tokyo, Japan \\ ${ }^{3}$ UEC, Advanced Wireless Communications research Center, Chofu Tokyo, Japan \\ ${ }^{4}$ Larboratoire de Physique et Chimie de l'Environnment et de l'Espace, Orléans, France
}

\begin{abstract}
The high-altitude ionospheric perturbations around the equatorial anomalies (EA) are often observed by DEMETER spacecraft on the nighttime orbits. The ionospheric perturbations are recognized as an enhancement of the electric field in the ELF frequency range in the satellite coordinate. Clear perturbations are mostly recognized on the dayside of the satellite orbit and the peak perturbation intensity tends to increase with increasing magnetic latitude based on around 1000 perturbation events observed in the year of 2005. The perturbation intensities are examined in relation with major seismic activities. As a result, most importantly higher perturbation intensity persists for the time periods around the occurrence days of the land earthquakes rather than those for the sea earthquakes and without major earthquakes nearby. However, the difference of the observed perturbation intensity between above-mentioned three cases are rather small due to relatively large variability indicative of the weak correlation of seismicity with the high altitude ionospheric perturbations near EA.
\end{abstract}

Key words: ionospheric perturbations, satellite observation, equatorial anomaly, seismic activity

\section{Introduction}

Variations of the ionospheric parameters in relation with the major seismic activity are the one of the key issues to understand the lithosphere-atmosphere-ionosphere coupling (LAIC) mechanism (e.g, a recent monograph edited by Hayakawa (2012)).

It is well known that the acoustic-gravity waves generated during and after the main shock perturb the ionosphere (e.g. Blanc, 1985, Calais and Minster, 1995). However the effect of seismic activity in the different altitudes of the ionosphere prior to the earthquake is still inconclusive. The perturbations in the bottom of the ionosphere (D-region) are monitored by the ground-based reception of the VLF transmitter signals. The D-region ionospheric perturbations before the major earthquakes are reported statistically (e.g. Hayakawa et al., 2010) and occur normally within one week before the main shock, while the perturbation in the F2 region of the ionosphere is found a few days before the earthquake with the methods such as ionospheric sounding stations and GPS TEC (Liu et al., 2000, 2001; Hobara et al., 2005a). Above the F2 region, such a kind of observation is available only by the spacecraft measurement.

Recently high altitude ionoshpheric perturbations for different spatial scales on the poleward side of the equatorial anomaly (EA) are identified by the satellite electric field measurement in the low frequency ranges. Short-scale $(0.1 \mathrm{~m}$ to $10 \mathrm{~m})$ density perturbation is identified by the electric field measurement in the VLF frequency range by IK-24 satellite (Molchanov et al., 2002), while the transitional scale (10-100m) are

Copyright 2013 by the Society of Atmospheric Electricity of Japan. 
statistically studied by the ELF electric field fluctuations by Aureol 3 satellite and the enhancements of the field intensity were found around the major seismic activities (Hobara et al., 2005b). Large scale electron density perturbations are directly measured by Cosmos-900 satellite and the decrease of the electron density was found in association with seismic activity (Molchanov et al., 2004).

However, the following problems still remain mainly due to the technical limitations such as (1) the satellite orbit used for previous studies (VLF/ELF) is not circular and is not able to sample the field at the same altitude and same local time, (2) the dynamic range of the instrument is not large enough to detect the EA related electric field perturbations because the primary purpose of the satellite is to observe the high latitudinal intensive phenomena, (3) wide-band spectrogram is not available (i.e. only few frequency components are observed by the filter bank system). Therefore even the data from several-year records do not provide a large amount of samples of the electric field perturbations. Recently the wide-band electric field data (ELF/VLF spectral density) available globally from the low-altitude French DEMETER satellite overcomes the above-mentioned limitations.

In this paper we analyze one-year continuous ELF electric field data recorded by the French DEMETER satellite at the high altitude ionosphere. The electric field perturbations presumably due to the high altitude ionospheric density perturbation are identified and are correlated with the land and sea earthquakes. As a statistical result the median peak intensity of the land earthquakes is slightly larger than those for sea earthquakes and no earthquakes both before and after the earthquakes. Despite rather large variability probably due to the high altitude of observations, it indicates the part of the LAIC.

\section{Data set and observations}

The DEMETER satellite has a circular and sun synchronous orbit with an altitude of about $660 \mathrm{~km}$ during the time period of the data analysis in 2006 . The measurements are made at about local day (10:30) and local night (22:30).

The electric field in the ELF range is acquired by the ICE experiment onboard the low orbit satellite DEMETER (Berthelier et al., 2006). DEMETER uses two different observation modes for electromagnetic waves; namely survey and burst modes. The electric field from the survey mode is used for our data analysis because of continuous coverage of the data over the globe. In the survey mode power spectral components of the electric field with a time resolution of $0.5 \mathrm{~s}$ or $2 \mathrm{~s}$ are obtained continuously above the entire globe except the polar region (from $-65^{\circ}$ to $+65^{\circ}$ latitudinal range) with one horizontal component perpendicular to the orbital plane The frequency range is from 0 $\mathrm{Hz}$ to $20 \mathrm{kHz}$ with a resolution of $19.53 \mathrm{~Hz}$. On the contrary, in the burst mode the electric field waveforms from three orthogonal components are sampled with a high frequency rate of $20 \mathrm{kHz}$ but are available only above the very limited region, which is not suitable for the purpose of this paper.

Figure 1 (a) shows a typical example of the electric power spectrogram during the half orbit of the DEMETER satellite for about 90 minutes for daytime. The color scale indicates the intensity of electric field. Two remarkable enhancements of the electric field intensity are identified around the frequency of $50 \mathrm{~Hz}$ with the magnetic latitude of $20^{\circ} \sim 40^{\circ}$ on the poleward side of the EA. These enhancements are symmetrical around the magnetic equator. As was reported in the previous works by Hobara et al. (2005b), the observed electric field fluctuations exhibit nearly an identical characteristic as upper ionospheric perturbations and are located on the side of the EA crest. 
Figure 1(b) indicates the mean electric field intensity by integrating over the frequency range from 0 to $100 \mathrm{~Hz}$ corresponding to the spectrogram in Figure 1(a). This integration process was made to efficiently extract information of the electric field fluctuations such as the peak intensity of the electric perturbation and corresponding geomagnetic latitude.

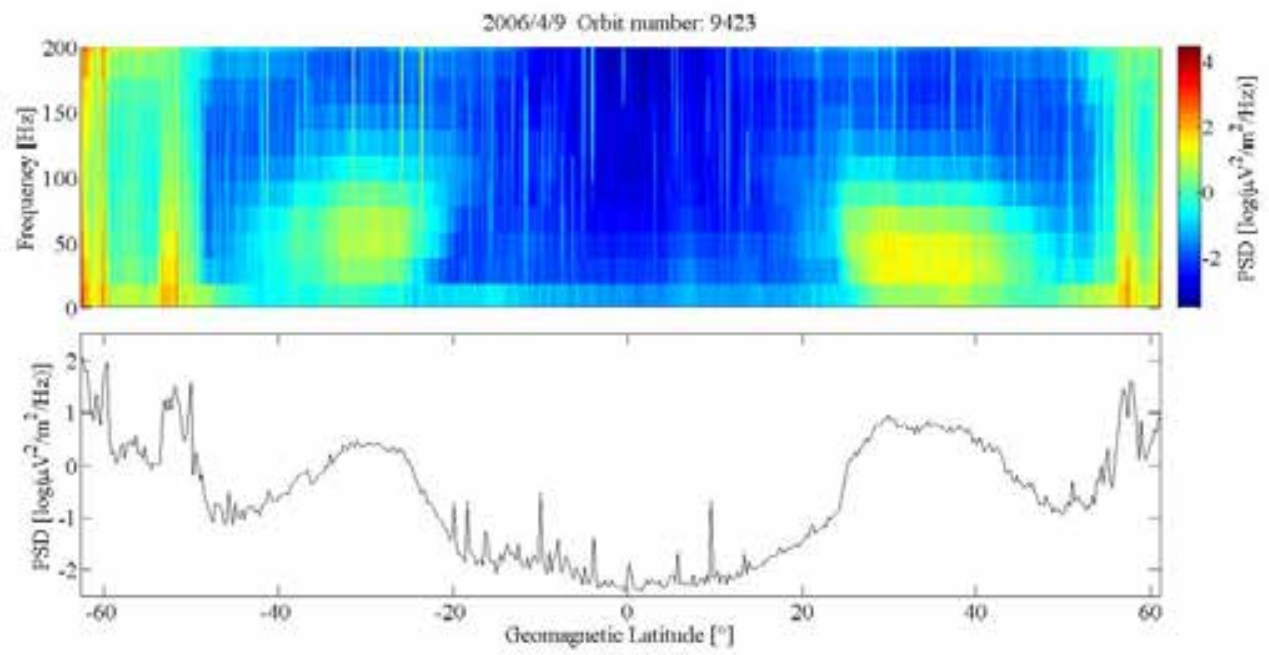

Figure 1 (a) Frequency spctrogram of the electric field observed by DEMETER for a half orbit, and (b) average electric field intensity in the ELF frequency range (from 0 to $200 \mathrm{~Hz}$ ) corresponding to the time period shown in (a).

The electric field enhancement near EA is mostly identified during daytime orbits in DEMETER. On the contrary, the electric field during nighttime orbits ( 22LT) mostly exhibits spiky nature and no clear persisting enhancement around EA latitude. Therefore the data during the local nighttime orbits are not used for our analysis.

\section{Observation results}

Monthly global maps of the integrated electric field intensity for dayside orbits are demonstrated in Figure 2. Figure 2 consists of 12 maps obtained for each month and is plotted in the coordinate system of geographic longitude (horizontal axis) and geomagnetic latitude (vertical axis). The color scale indicates the intensity of the integrated electric field from 0 to $100 \mathrm{~Hz}$. As is seen from the figure, the electric field intensity is the lowest in the magnetic equator and increases with increasing magnetic latitude as a general trend. In the mid latitude region, remarkable enhancements of the electric field are observed along the selected orbits. These enhancements occur at the latitude around the outer side of the EA as is indicated in Figure 1 and are often symmetrical about the magnetic equator. Moreover occurrences of these perturbations do not have a clear seasonal dependence. In the high latitude region $\left(>45^{\circ}\right)$, remarkable increase of the electric field is seen. These enhancements correspond to the intensive electrostatic turbulence in association with the field-aligned density enhancement with ELF hiss (Cerisier et al., 1985). 

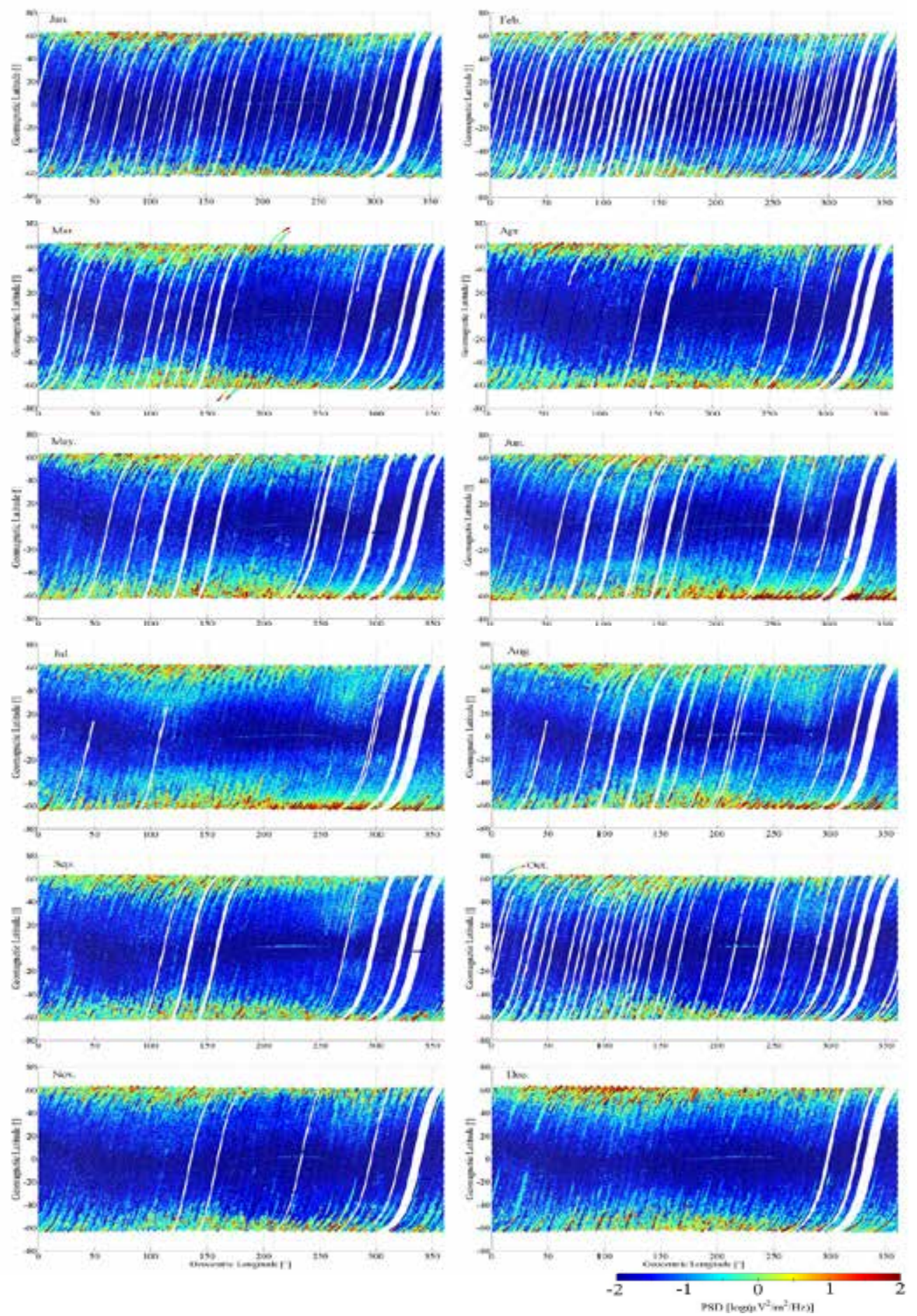

Figure 2 Monthly global distributions of the averaged electric field intensity in the ELF frequency range observed by DEMETER in the year of 2006. 
Figure 3 shows the global distribution of major earthquakes associated with electric field enhancement in the year of 2006 obtained from the USGS. The earthquakes used for our analysis satisfy the following conditions, (1) magnitude $\mathrm{M} \geq 6$ and (2) depth $\mathrm{D}<40 \mathrm{~km}$. The diameter of the circle corresponds to the magnitude of each earthquake. Furthermore the earthquakes are spatially separated by the land and undersea. The sea earthquakes are defined by the hypocenter reasonably far $(>100 \mathrm{~km})$ from the nearest cost. In Figure 3 , the red and blue points respectively indicate the earthquakes occurred on land and undersea. As is seen from the figure, the locations of the analyzed earthquakes correspond to major seismic regions but are not distributed at particular regions over the globe. This is important for further statistical analysis of the data.

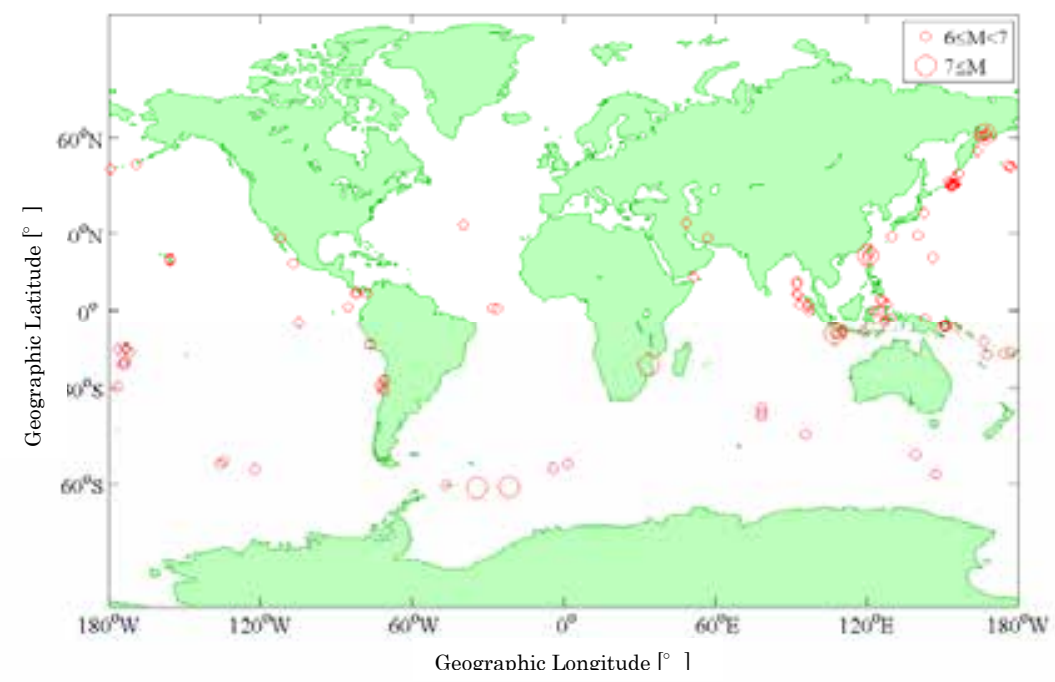

Figure 3 Global distributions of the major seismic activities associated with the ionospheric perturbation. The circles indicate the earthquake epicenters.

Figure 4 shows the latitudinal dependence of the peak electric field intensity for the electric field perturbations between 10 days prior to and the day of the earthquakes in 2006 (i.e. earthquakes occurred from 10 to 0 day before the turbulences). The absolute magnetic latitude including both hemispheres is used to demonstrate the latitudinal dependence because the occurrence locations of the perturbations are correlated well with EA which is symmetric about the magnetic equator for both hemispheres rather than the geographic latitude (Figure 2). Indeed the intensity distributions for both northern and southern hemispheres in the magnetic latitudes exhibit very similar nature.

In Figure 4 the filled circles indicate the turbulence associated with the land earthquakes, while inverted triangles indicate those associated with sea earthquakes. Triangles show that the perturbations no association with earthquakes according to our criteria (i.e. at least no major seismic events occurred between the 0 day and 10 days of the perturbation). The numbers of perturbations are large enough to perform the statistical analysis (40 perturbations for land earthquakes, 67 perturbations for sea earthquakes and 440 perturbations for no seismic events). Although the peak intensity tends to increase with increasing the magnetic latitude, the distribution has rather scattered nature and so the median with upper and lower quartiles are calculated for $5^{\circ}$ interval of the magnetic latitude from $25^{\circ}$ to $40^{\circ}$. 


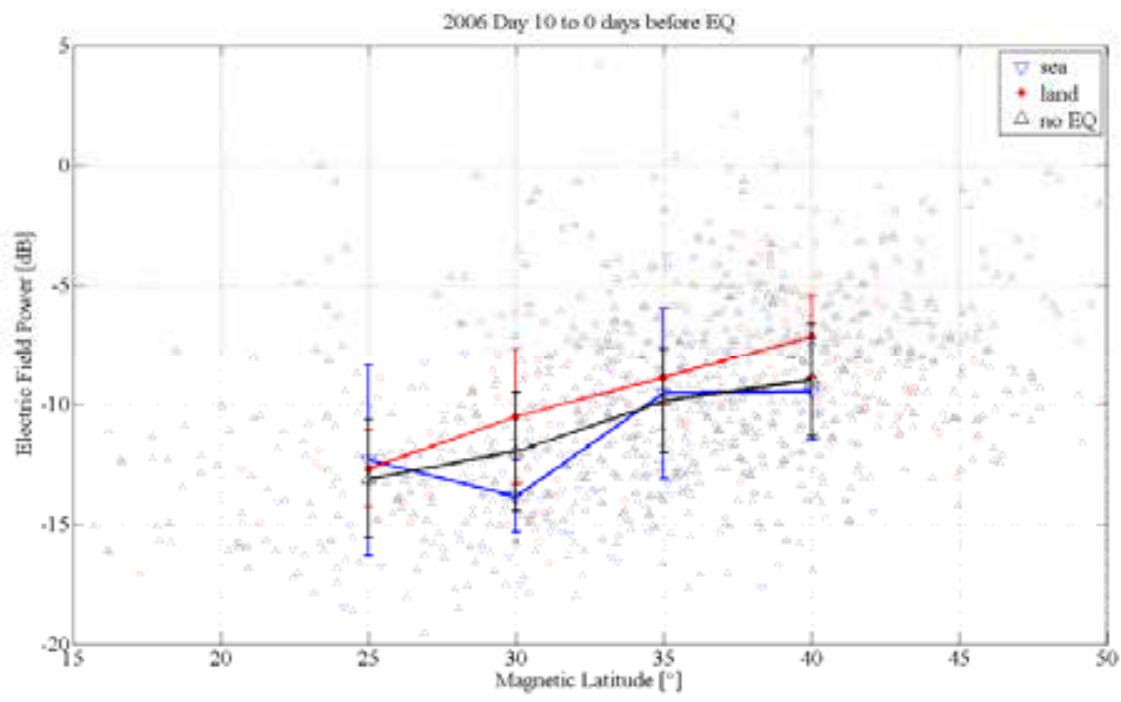

Figure 4 Latitudinal dependence of the ionospheric perturbation intensity for nighttime orbits in the year of 2005. Filled circles and inverted triangles indicate the perturbation intensity related to the land and sea earthquakes, respectively. The earthquakes occurred from 10 to 0 day before the turbulences (i.e. the ionospheric turbulences occur after the earthquakes). Triangles show the case of perturbation with no earthquakes.

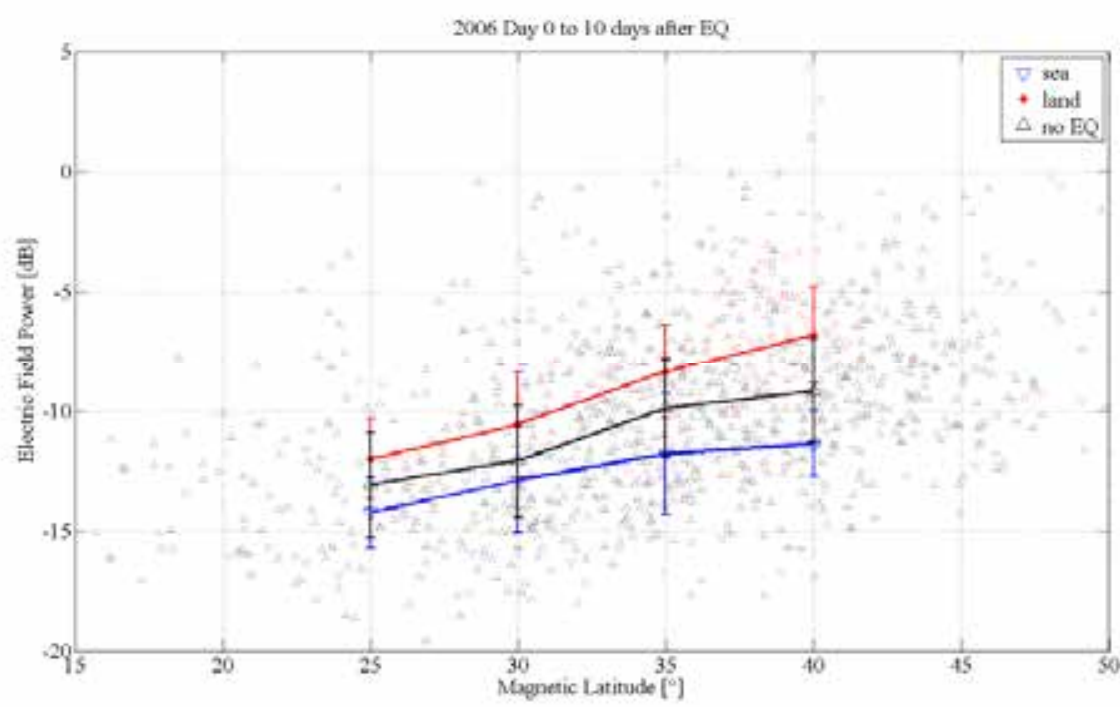

Figure 5 Same type of variation as Figure 4 but for the earthquakes occurred from 0 to 10 days after the turbulences (i.e. the ionospheric turbulences occur before the earthquakes). 
As is seen from Figure 4, the median of the peak electric field perturbations increases monotonically with increasing magnetic latitude for all three categories (before the land earthquakes, sea earthquakes and no earthquake). The variability of the intensity at different latitudes defined by upper and lower quartiles from the median values (the vertical lines in Figure 4) is quite large for all latitudinal intervals and so the difference in peak intensity between three categories is not statistically significant. However, most importantly the median peak electric intensity for the land earthquakes is higher than other two categories except $25^{\circ}$ (almost the same values for three cases).

Figure 5 shows the same type of plot with Figure 4 but for the electric field perturbations after the earthquakes in 2006 (i.e. turbulence occurred from 0 to 10 days after the earthquake). The numbers of perturbations are again large enough to perform the statistical analysis (70 perturbations for land earthquakes, 27 perturbations for sea earthquakes and 428 perturbations for no seismic events). The median values of the peak intensity for land earthquakes increases with increasing the magnetic latitude and are the largest among the three categories as is seen for the perturbations before the earthquakes (Figure 4). The magnitude of the peak electric field intensity for the land earthquakes is also quite similar. One remarkable difference is that the intensity for sea earthquakes is the smallest among the three categories for all latitudinal intervals.

\section{Discussion and summary}

In this paper we perform the similar data analysis as was reported by Hobara et al. (2005b) by using the ELF electric field data from Aureol 3 satellite. They found that mean value of the electric field power during the seismic period is much higher than that for no seismic period. However the electric field data from Aureol satellite obtained by the filter banks (only 3 frequency channels below $100 \mathrm{~Hz}$ ) are not of high sensitivity over the EA region. Moreover, the orbit is not elliptical and so a small number of points for the data analysis were available around the perigee. Hence the land and sea earthquakes were not separated. Therefore the data used in this study by DEMETER certainly has advantages for above-mentioned points such as continuous survey frequency spectra, rather high sensitivity of the instrument, and complete coverage of the globe thanks to the low altitude circular orbit.

The newly obtained results show that the peak electric field perturbation intensity has a clear latitudinal dependence with symmetry about the magnetic equator but has a large variability. And the statistical parameters are used to evaluate the spatial characteristics of the perturbation such as median, upper and lower quartiles. The perturbation intensity tends to increase with increasing magnetic latitude which could be related to the latitudinal dependence of the instability process of the redistributed plasma forming the EA. In the observed frequency range (ELF) and the altitude $(500 \sim 700 \mathrm{~km})$, the $\boldsymbol{k}$ distribution of the plasma density perturbations is similar to the $\boldsymbol{k}$ spectrum of the electric field intensity (Molchanov et al., 2004). Although EA is the daily process, the perturbations in this paper were observed only under the particular conditions such as some kind of forcing process by e.g. atmospheric gravity wave (AGW).

Being different from the similar types of previous reports (e.g. Molchanov et al., 2004; Hobara et al., 2005b), the spatial and temporal dependences of the seismicity are separately treated in this paper. However differences between the seismic and non-seismic cases is not statistically proved in our results because in most cases the large variability indicated by significant overlapping error bars (upper and lower quartiles between the cases) are seen for different latitude ranges. However there are certain tendencies in the median values despite large variability, which makes following 
remarks.

The median electric field perturbation intensity in relation with the land earthquakes is stronger than that from no-seismic and sea earthquakes for all magnetic latitude considered in this paper. And the latitudinal dependence of the intensity does not change relative to the occurrence time of the earthquakes (i.e. before and after earthquakes), which indicates that the effect of the seismicity starts before the main shock and persists for several days even after the earthquake. However the intensity for the sea earthquakes has a similar value as the perturbation without earthquakes, the intensity decreases after the earthquake below the no seismic cases or there is no effect at all. It could be due to the nature of the sea earthquakes such as the forcing mechanism of the precursor acting more prominent (e.g. more effectively penetrating toward the sea surface from the hypocenter) before the earthquakes. The possible hypotheses linking to the ionospheric perturbations from the seismicity are proposed such as electric field anomaly due to the geochemical effects (Pulinets et al., 1994) and thermal anomaly (Tramutoli et al., 2001; Tronin et al., 2002) leading to the AGW (Molchanov and Hayakawa, 2008; Hayakawa, 2009). Although the preferred mechanism to generate the perturbation near the EA observed by this paper is AGW hypothesis (Mareev et al., 2002), more careful consideration is highly required.

Above-mentioned experimental results indicate that the effect of the seismicity toward the electric perturbation corresponding to the density perturbation at the observed altitude $(650 \mathrm{~km})$ may be too small to be proved statistically. However the weak correlation with land earthquake exists as persisting increased perturbation intensity starting before seismic activity, which is indicative of the LAIC. Further study is necessary to elucidate quantitatively the coupling mechanism by comparing the measurements from different altitudes.

Acknowledgement. The authors would like to thank J. J. Berthelier for ICE electric field data and are grateful to the Guest Investigator program by CNES for the DEMETER mission. This work is partially supported by Special Management Expenses Grants for National University Corporations (for Projects) from the Ministry of Education, Culture, Sports, Science and Technology of Japan (MEXT).

\section{References}

Berthelier, J.J., et al., ICE, the electric field experiment on DEMETER, Planet. Space Sci., 54, 456-471, doi:10.1016/j.pss.2005.10.016, 2005.

Blanc, E., Observations in the upper atmosphere of infrasonic waves from natural or artificial sources: A summary, Ann. Geophysicae, 3, 673-679.1985.

Calais, E. and J.B. Minster, GPS detection of ionospheric perturbations following the January 17, 1994, Northridge earthquake, Geophys. Res. Lett., 22, 1045-1048, 1995.

Cerisier, J. C., J.J. Berthelier and C. Béghin, Unstable density gradients in the high-latitude ionosphere, Radio Sci., 20, 755-761, 1985.

Hayakawa, M. (Ed), The Frontier of Earthquake Prediction Studies, Nihon-senmontosho-Shuppan, Tokyo, 794p, 2012.

Hayakawa, M., Lower ionospheric perturbations associated with earthquakes, as detected by subionospheric VLF/LF radio signals, in "Electromagnetic Phenomena Associated with Earthquakes", Ed. by M. Hayakawa, Transworld Research Network, Trivandram (India), 137-185, 2009.

Hayakawa, M., Y. Kasahara, T. Nakamura, F. Muto, T. Horie, S. Maekawa, Y. Hobara, A. 
A., Rozhnoi, M. Solivieva and O. A. Molchanov, A statistical study on the correlation between lower ionospheric perturbations as seen by subionospheric VLF/LF propagation and earthquakes, J. Geophys. Res., vol. 115, A09305, doi:10. 1029/2009JA015143, 2010.

Hobara, Y. and M. Parrot, Ionospheric perturbations linked to a very powerful seismic event, J. Atmos. Solar-Terr. Phys., 67, 677-685, 2005a.

Hobara, Y., F. Lefeuvre, M. Parrot and O.A. Molchanov, Low-latitude ionospheric turbulence observed by Aureol-3 satellite, Ann. Geophysicae, 23, 1259-1270, 2005b.

Liu, J.Y., Y.I. Chen, Y.J. Chuo and H.F. Tsai, Variations of ionospheric total electron content during the Chi-Chi earthquake, Geophys. Res. Lett., 28, 1383-1386, 2001.

Liu, J.Y., Y.I. Chen, S.A. Plinets, Y.B. Tsai and Y.J. Chuo, Seismo-ionospheric signatures prior to MX6.0 Taiwan earthquakes, Geophys. Res. Lett., 27, 3113-3116, 2000.

Mareev, E.A., D.I. Iudin and O.A. Molchanov, Mosaic source of internal gravity waves associated with seismic activity. In: Hayakawa, M., Molchanov, O.A. (Eds.), Seismo-Electromagnetics: Lithosphere-Atmosphere-Ionosphere Coupling, TERRUPUB, Tokyo, pp. 335-343, 2002.

Molchanov, O.A. and M. Hayakawa, Seismo-Electromagnetics and Related Phenomena: History and latest results, TERRAPUB, Tokyo, 189p, 2008.

Molchanov, O.A., M. Hayakawa, V.V. Afonin, O.A. Akentieva and E.A. Mareev, Possible influence of seismicity by gravity waves on ionospheric equatorial anomaly from data of IK-24 satellite, 1. Search for idea of seismo-ionosphere coupling; 2. Equatorial anomaly and small-scale ionospheric turbulence. In: Hayakawa M. and O.A. Molchanov (Eds.), Seismo-Electromagnetics: Lithosphere-Atmosphere-Ionosphere Coupling, TERRUPUB, Tokyo, pp. 275-297. 2002.

Molchanov, O.A., O.S. Akentieva, V.V. Afonin, E.A. Mareev and E. Fedorov, Plasma density-electric field turbulence in the low-latitude ionosphere from the observation on satellites; possible connection with seismicity, Phys. Chem. Earth, 29, 569-577, 2004.

Pulinets, S. A., A. D. Legen'ka and V. A. Alekseev, Preearthquakes, effects and their possible mechanisms, In: Dusty and Dirty Plasmas, Noise and Chaos in Space and in the Laboratory, Ed. by H. Kikuchi, Plenum Publishing, New York, pp. 545-557, 1994.

Tramutoli, V., G. Di Bello, N. Pergola and S. Piscitelli, Robust satellite techniques for remote sensing of seismically active areas, Annali de Geofisica, 44, 295-312, 2001.

Tronin, A. A., Atmosphere-lithosphere coupling. Thermal anomalies on the Earth surface in seismic processes, In: Hayakawa, M. and O.A. Molchanov (Eds.), Seismo-Electromagnetics: Lithosphere-Atmosphere-Ionosphere Coupling, TERRAPUB, Tokyo, pp. 173-176, 2002.

(Received November 13, 2012; revised November 16, 2012; accepted November 20, 2012) 